\title{
Estudo dos Índices de Impulsividade e Cauda-Pesada de Redes sem Fio com Tecnologia IEEE 802.11
}

\author{
L. Zão, M.C. Diniz e R. Coelho
}

\begin{abstract}
Resumo-Este trabalho apresenta um estudo de estimação da impulsividade em redes sem fio. A representação da impulsividade foi examinada e discutida considerando-se diferentes processos estocásticos (fBm, $M / G / \infty$ e Pseudo-MMPP). Os resultados de estimação demonstraram que o tráfego de redes sem fio apresenta alto grau de impulsividade. De forma geral, os modelos fBm e $M / G / \infty$ obtiveram interessante representação da impulsividade. $O$ comportamento da impulsividade considerando-se processos de chegadas com diferentes índices e distribuição de cauda-pesada também foi avaliada em filas.
\end{abstract}

Palavras-Chave-Redes sem-fio, impulsividade, processos alpha-stable, distribuições cauda-pesada.

Abstract-This paper presents a wireless traffic impulsiveness estimation analysis. The impulsiveness degree representation is also examined for different heavy-tail stochastic processes (fBm, $M / G / \infty$, and Pseudo-MMPP). The impulsiveness behavior on queueing systems was also investigated considering different degrees and heavy-tail distributions.

Keywords-Wireless traffic, impulsiveness degree, alpha-stable processes, heavy-tail distribution.

\section{INTRODUÇÃO}

As redes sem fio baseadas na tecnologia IEEE802.11 [1] vêm se popularizando como meio de comunicação em redes acadêmicas, corporativas e públicas. Atualmente, prédios ou departamentos de uma mesma empresa ou universidade são facilmente conectados por estas redes sem fio, sem que haja a necessidade de infra-estrutura para passagem de cabos e sem exigência de localização fixa para os usuários. A representação do perfil do tráfego de usuários em pontos de acesso a redes sem fio é um importante desafio para o dimensionamento e a implantação dos futuros sistemas sem fio.

Diferentes estudos procuraram caracterizar o perfil do tráfego de usuários de redes sem fio. Basicamente, esses trabalhos usaram 3 traces disponíveis publicamente: o trace UCSD [2] que representa o uso de uma rede sem fio durante os 3 dias da conferência ACM/SIGCOMM; os traces Darthmouth [3] que apresentam o tráfego da rede de um campus universitário com 1706 usuários espalhados em 161 prédios e com 476 pontos de acesso; e o trace IBM [4] que representa uma rede sem fio corporativa espalhada por 3 prédios da empresa com 1366 usuários e 174 pontos de acesso.

Em [5], os autores analisaram o trace da conferência ACM/SIGCOMM e identificaram presença de dependência de

L. Zão e R. Coelho, Instituto Militar de Engenharia, M.C. Diniz, Universidade Federal do Estado do Rio de Janeiro, Rio de Janeiro, Brasil, Emails:zao@ime.eb.br, coelho@ime.eb.br, morganna@uniriotec.br. Este trabalho foi parcialmente financiado pelo CNPq, FINEP e FAPERJ. longo alcance (Long-Range Dependence - LRD) [6]. Em [7], os autores monitoraram uma rede sem fio por rádio da cidade de Vancouver (Canadá) para atendimentos à emergências com 2161 usuários (policiais, bombeiros, ambulâncias e trânsito) por 7 semanas. Um resultado interessante, mostrou que os tempos entre chamadas eram exponencialmente distribuídos mas exibiam um certo grau de LRD.

Em [9], estudou-se o comportamento dos usuários da rede sem fio da Universidade da Carolina do Norte e é feita a comparação com o comportamento dos usuários dos traces de Darthmouth. Em [10], é mostrado que o tráfego 802.11 agregado é melhor modelado com um processo multifractal.

É importante observar que nem todos os traces utilizados nos trabalhos publicados são de domínio público. Este é o caso dos traces usados em [7] e [9]. Além disso, não é possível usar alguns traces para a caracterização do tráfego de redes sem fio. É o caso dos traces de Darthmouth [3] que apresentam variância infinita em relação a quantidade de bytes/pacotes transmitidos.

Em um estudo anterior [8], foi apresentada a caracterização de tráfegos de pacotes em pontos de acesso de redes sem fio baseado em modelos pseudo-markovianos e fractais. Processos estocásticos com distribuição de cauda-pesada apresentam alto grau de impulsividade ou variância infinita [11] [12]. Esta impulsividade é atribuída ao lento decaimento da cauda em comparação a distribuição Gaussiana. A impulsividade dos processos HTD (Heavy-Tail Distribution), é mantida mesmo após a agregação de inúmeras ordens do processo ou fontes de tráfego. Isso dificulta a análise da multiplexação de fluxos uma vez que esta geralmente é baseada na teoria de convergência da distribuição Normal definida pelo teorema do limite central. Se um processo estocástico $X(t)$ possui HTD $(P[X(t)>x])$, sua convergência é representada por um processo Alpha-Stable com variância infinita e $1<\alpha<2$. Por definição, $0<\alpha<2$. Contudo processos com os valores de $\alpha<1$ possuem média infinita. Um outro grande limitante dos estudos baseados nos processos Alpha-Stable [13], concerne a complexidade da estimação de seus parâmetros básicos. Isso se deve a sua intrínseca impulsividade (variância infinita) e também pelo fato de $\alpha=2$ (processo Gaussiano) ser o limitante ou a discontinuidade dos processos Alpha-Stable. Os processos Alpha-Stable são também denominados na literatura por nãoGaussiano. Assim sendo, o estudo das características de impulsividade e cauda-pesada são de fundamental importância para o dimensionamento dos pontos de acesso das redes de sem fio. 
Os principais objetivos deste trabalho são portanto: estimar a impulsividade do tráfego de pacotes em redes sem fio, analisar o desempenho dos modelos HTD movimento Browniano fracionário (fBm-fractional Brownian motion) [14], $M / G / \infty$ [15] e Pseudo-MMPP [16] para representação da impulsividade e avaliar a precisão das medidas de desempenho considerando filas para processos de chegadas com distribuição com cauda-pesada.

O restante deste trabalho está organizado da seguinte forma. A seção II apresenta resultados de estimação de impulsividade das redes sem fio. A seção III apresenta uma descrição sucinta dos modelos HTD analisados para representação de tráfego com impulsividade. A seção IV discute os resultados obtidos com o estudo dos traces. Finalmente, a seção V apresenta as principais conclusões deste artigo.

\section{EstimaÇÃO DA IMPULSIVIDADE EM REDES SEM FIO}

A impulsividade de um determinado sinal ou tráfego pode ser representada pela família de distribuições Alpha-Stable (AS) [12] [11]. Alguns casos particulares desta família são as distribuições Gaussiana, Cauchy e Lévy que possuem impulsividade definida por $\alpha=2, \alpha=1$ e $\alpha=1 / 2$, respectivamente. Processos com valores de $\alpha<1$ apresentam média infinita.

Variáveis aleatórias (V.A.) AS devem obedecer a propriedade de estabilidade [11][17], ou seja, se $X, X_{1}$ e $X_{2}$ são V.As independentes e identicamente distribuídas (i.i.d.) então existem $\mu_{1}$ e $\mu_{2}$ que satisfazem a expressão:

$$
r_{1} X_{1}+r_{2} X_{2} \stackrel{d}{\cong} \mu_{1} X+\mu_{2}
$$

onde $r_{1}, r_{2}, \mu_{1}$ e $\mu_{2}$ são constantes e $\stackrel{d}{\cong}$ significa semelhante em distribuição. Além disso, devem também satisfazer o teorema do limite central onde $X$ será AS se somente se

$$
S_{n}=\frac{X_{1}+X_{2}+\ldots+X_{n}}{a_{n}}-b_{n}
$$

onde $X_{1}, X_{2}, \ldots$ são V.As i.i.d. e $n \rightarrow \infty, a_{n}$ e $b_{n}$ são parâmetros reais, sendo $a_{n}$ positivo.

A função característica $(\phi(\theta))$ de uma V.A. da família AS é definida por $\phi(\theta)=\phi(j v X)=\exp \left(-\sigma^{\alpha}|v|^{\alpha}\right)$ onde $\alpha$ é o expoente ou índice de $\phi(\theta), \beta$ define a localização e $\gamma=\sigma^{\alpha}$ é o parâmetro de escala ou dispersão da variável aleatória. Um processo AS é completamente definido por 3 parâmetros: $\alpha$ $(0<\alpha<2), \beta(-1<\beta<1)$ e $\gamma(0, \infty)$. Processos $\operatorname{com} \beta=$ 0 são denominados simétricos (SAS, Symmetric Alpha-Stable) e estes são geralmente adotados nos estudos de estimação e modelagem.

Devido as características dos processos SAS, a estimação de seus parâmetros é altamente complexa. Os principais métodos de estimação apresentados na literatura foram propostos por McCulloch [18], Kogon [19], Ma [20] e Tsihrintzis [21]. Uma detalhada análise comparativa desses algoritmos é feita em [22] que apresenta duas conclusões importantes. Em primeiro lugar, o método McCulloch, baseado na estatística de ordem fracionária [12] de uma seqüência amostral, é o algoritmo mais completo (atingiu estimação dos 3 parâmetros) e preciso. Em segundo lugar, quando os valores dos métodos divergem muito entre si, isto significa que os dados não compõem uma distribuição SAS.

A estimação dos parâmetros de distribuições SAS ainda é um importante desafio para as pesquisas da área e para a modelagem de tráfego com presença de impulsividade. Segundo [23], erros de medidas de desempenho da ordem de $100 \%$ podem ocorrer se o processo SAS tiver, por exemplo, valores $\alpha<1,7$. Quando os valores dos métodos divergem muito entre si, isto significa que os dados não compõem uma distribuição SAS.

Objetivando estudar a impusividade em redes sem fio, foram analisados os traces IBM. Estes traces foram escolhidos por serem os únicos, disponíveis na Internet, que possuem todos dados necessários a este estudo. A coleta dos dados foi feita em 3 diferentes prédios denominados em [4] de SBldg (Small Building), MBldg (Medium Building) e LBldg (Large Building). Esta mesma nomenclatura foi adotada neste trabalho.

A Tabela I mostra a estimação do parâmetro $\alpha$ para os 174 pontos de acesso estudados (tráfego de pacotes entrada e saída). Note que a maioria dos pontos de acesso possui valor de $\alpha$ concentrado principalmente na faixa que vai de 0,5 a 1 . Ou seja, o tráfego da rede sem fio possui alta impulsividade. Lembre também, que valores $\alpha<1$, são representantes de média infinita, e segundo [23], a probabilidade de erro de precisão de $100 \%$ para processos SAS com $\alpha<1,7$ não é desprezível $(\approx 0,03)$. Em [17], os autores também identificaram impulsividade $(\alpha=1,55)$ em tráfego coletado em bytes na rede 802.11 b local da Universidade de Calgary, Canadá.

\begin{tabular}{|c|c|c|}
\hline$\hat{\alpha}$ & PA (Tráfego Entrada) & PA (Tráfego Saída) \\
\hline $0,5<\hat{\alpha}<=1,0$ & 136 & 119 \\
\hline $1,0<\hat{\alpha}<=1,5$ & 16 & 28 \\
\hline $1,5<\hat{\alpha}<=1,7$ & 0 & 0 \\
\hline $1,7<\hat{\alpha}<=2,0$ & 22 & 27 \\
\hline
\end{tabular}

TABELA I

QuANTIDADE DE PAS SEGUNDO O VALOR DE $\alpha$ PARA TRÁFEGo DE ENTRADA E SAÍDA.

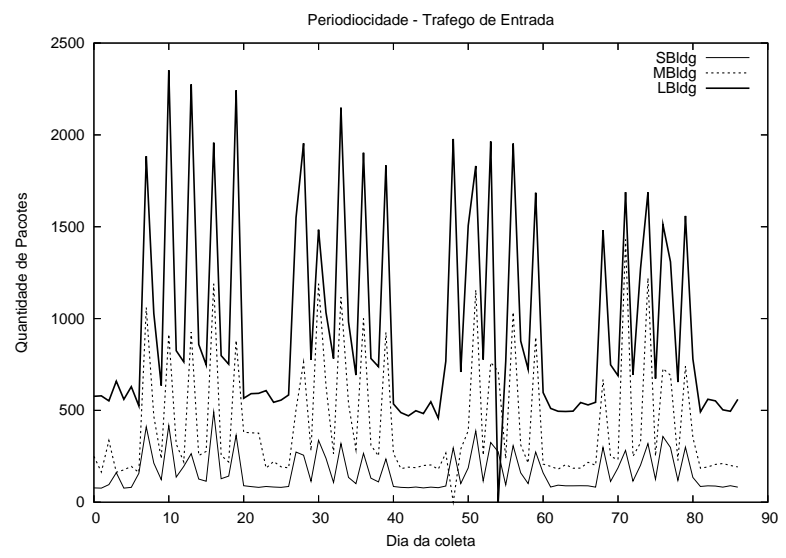

Fig. 1. Tráfego de entrada de diferentes pontos de acesso

Em [8] e [24], o estudo dos traces de vários pontos de acesso de redes sem fio mostrou que o tráfego possui 


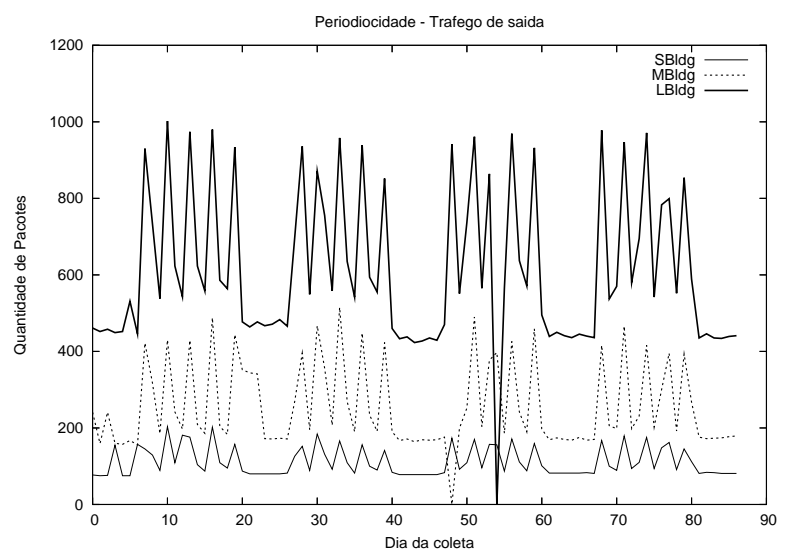

Fig. 2. Tráfego de saída de diferentes pontos de acesso

perfil padrão semelhante para os diversos dias de coleta considerando-se os diferentes prédios. Esta característica pode ser observada nas Figuras 1 e 2. Estas figuras representam a quantidade de pacotes transmitidos nos 3 prédios durante os 90 dias da coleta. Considerando os comentários acima, nesse trabalho foram selecionados, aleatoriamente, um trace de cada prédio com $\alpha$ entre 1,7 e 2 .

\section{REPRESENTAÇÃO DA IMPULSIVIDADE POR PROCESSOS HTD}

A HTD ou função distribuição complementar de um processo estocástico $X(t)$ é definida por $P[X(t)>x] \cong$ $c x^{-\alpha}, \quad x \rightarrow \infty$, sendo $c$ uma constante positiva e $\alpha \in$ $(0,2)$. Se um processo possui cauda, então o seu decaimento não é exponencial (característica dos processos SRD). Este comportamento dos processos HTD define o grau de impulsividade de um processo estocástico. Quanto mais pesada ou longa for a cauda, maior será a impulsividade $(\alpha<2)$. Esta impulsividade ou não-convergência, pode ser também verificada pelo grau de dependência temporal do processo. $\mathrm{O}$ grau de dependência temporal ou simplesmente parâmetro de Hurst $(H)$ de um processo estocástico, pode ser definido pela taxa de decaimento da função auto-correlação (FAC), $\rho(k)$, do processo quando $k \rightarrow \infty$. fBm, $M / G / \infty$ e Pseudo-MMPP são importantes modelos de representação de distribuição HTD.

\section{A. $f B m$}

O movimento Browniano fracionário [14] é um processo estocástico Gaussiano $\left(X_{H}(t)\right)$ de parâmetro contínuo $t$, média nula e incrementos estacionários, independentes, cuja variância é proporcional ao intervalo de tempo entre eles. O fBm é um processo auto-similar cujo grau de dependência é definido pelo parâmetro $H$. Isto significa que suas características estatísticas se mantêm para qualquer escala no tempo. Assim, para quaisquer $\tau$ e $r>0$ temos que:

$$
\left[X_{H}(t+\tau)-X_{H}(t)\right]_{\tau \leq 0} \stackrel{d}{\approx} r^{-H}\left[X_{H}(t+r \tau)-X_{H}(t)\right]_{\tau \leq 0}
$$

onde $r$ é o fator de escala do processo e o símbolo $\stackrel{d}{\approx}$ significa semelhante em distribuição. Um processo fBm, $X_{H}(t)$, é completamente caracterizado por sua média $(m)$, variância $\left(\sigma^{2}\right)$ e $H$ e sua FAC é definida por

$$
\rho(k)=\frac{1}{2} \sigma^{2}\left[(k+1)^{2 H}-2 k^{2 H}+(k-1)^{2 H}\right] .
$$

Em [25], os autores propuseram a discretização do fBm, obtendo um novo processo $A(t)$ com características de invariância em escala, mas com média e variância não-nulas. Tomando, por exemplo, $A(t)$ como sendo o número de bits recebidos por um ponto de acesso da rede sem fio até o instante $t$, temos que

$$
A(t)=m t+\sqrt{a m} X_{H}(t),
$$

onde $m$ é a taxa média de chegadas de bits, $a=$ $\operatorname{Var}[A(t)] /(m t)^{2 H}$ é o coeficiente de variância e $H \in\left(\frac{1}{2}, 1\right)$ é o parâmetro $H$.

\section{B. $M / G / \infty$}

O modelo $M / G / \infty$ foi proposto em [15] com o principal objetivo de representar processos SRD $\left(H=\frac{1}{2}\right)$ e LRD $\left(H>\frac{1}{2}\right)$. O modelo é representado por um servidor infinito com chegada Poisson de taxa média $\lambda$ e tempo de serviço com distribuição $G$. Nesta proposta a representação da LRD é garantida através da definição de $G$ como uma distribuição de cauda-pesada. A distribuição $G$ do processo estocástico representante do tempo de atendimento $Z(t)$ do modelo $M / G / \infty$ deve obedecer a seguinte relação:

$$
P[Z>t]=\frac{\Psi(t)-\Psi(t+1)}{1-\Psi(1)}, \quad t=0,1, \ldots
$$

onde $\Psi(t)$ é a FAC do processo $Z(t)$. O modelo $M / G / \infty$ é completamente definido por $(\lambda, G)$. Os autores mostraram que a FAC de uma seqüência LRD é melhor modelada por

$$
\rho(k)=e^{-\beta \sqrt{k}}, \quad k=0,1,2, \ldots
$$

onde o parâmetro $\beta$ é estimado a partir do trace original. Através da adequada escolha da distribuição $G$, e conhecendose o valor de $\beta$ (valor estimado do trace original), conseguese obter a FAC da Eq.5 para $\frac{1}{2}<H<1$. A FAC do processo $Z(t)$ está relacionada à covariância $(\Gamma()$.$) por \Psi(t)=$ $\frac{\Gamma(t)}{\Gamma(0)}, t=0,1,2, \ldots$ A covariância do $M / G / \infty$ é definida por $\Gamma(h)=\delta^{2} \Psi_{H}(t), h=0,1, \ldots$, onde $H=1-\beta / 2$ e $\beta$ $(0<\beta<1)$ e $\delta^{2}>0$ são constantes. Para conseguir a representação do grau de dependência positivo $(H>1 / 2)$, o processo $M / G / \infty\left(Z_{H}(t)\right)$ deve ter uma FAC decrescente $\left(\Psi_{H}(t)\right) \operatorname{com} \Psi_{H}(0)=1$. Então,

$$
\Psi_{H}(t) \sim H(2 H-1) t^{2 H-2}, \quad t \rightarrow \infty
$$

Para encontrar a distribuição do processo $Z_{H}(t)$ definido pela FAC da Eq. 6, temos que:

$P\left[Z_{H}>z\right]=\frac{|z+2|^{2 H}-3|z+1|^{2 H}+3|z|^{2 H}-|z-1|^{2 H}}{4\left(1-2^{2 H-2}\right)}$,

onde $z=1,2, \ldots$ Esse processo terá característica de dependência representada pelo parâmetro $H$. A distribuição $G$ é relacionada à FAC pela expressão:

$$
P[Z=k]=\frac{\rho(k-1)-2 \rho(k)+\rho(k+1)}{1-\rho(1)} .
$$




\section{Pseudo-MMPP}

Em [16], os autores propuseram alcançar a LRD através da sobreposição de diversos processos MMPPs (MarkovModulated Poisson Process) de dois estados. Esta proposta é aqui denotada por Pseudo-MMPP. A sobreposição de MMPPs é representada pela sobreposição de IPPs (Interrupted Poisson Process) e de um processo Poisson. Cada IPP é um processo de 2 estados onde as chegadas ocorrem apenas em um dos estados (chamado de ativo) de acordo com uma distribuição Poisson.

Suponha que $Q_{i}$ e $\Lambda_{i}$ sejam, respectivamente, a matriz de transição dos estados e a matriz de taxas de chegadas do $i$ ésimo IPP, então

$$
Q_{i}=\left[\begin{array}{cc}
-q_{1 i} & q_{1 i} \\
q_{2 i} & -q_{2 i}
\end{array}\right] \text { e } \quad \Lambda_{i}=\left[\begin{array}{cc}
\lambda_{i} & 0 \\
0 & 0
\end{array}\right] .
$$

A sobreposição de $d$ MMPPs pode ser descrita como

$$
\begin{gathered}
Q=Q_{1} \oplus Q_{2} \oplus \cdots \oplus Q_{d} \quad \mathrm{e} \\
\Lambda=\Lambda_{1} \oplus \Lambda_{2} \oplus \cdots \oplus \Lambda_{d} \oplus \lambda_{p},
\end{gathered}
$$

onde $\oplus$ corresponde a soma de Kronecker e $\lambda_{p}$ é a taxa de chegadas do processo Poisson. Logo, a taxa total de chegadas do modelo pode ser obtida por

$$
\lambda=\lambda_{p}+\sum_{i=1}^{d} \frac{q_{2 i}}{q_{1 i}+q_{2 i}} \lambda_{i} .
$$

É importante observar que a variância do tráfego não é considerada pelo algoritmo de Andersen e Nielsen na escolha dos parâmetros dos processos MMPPs.

\section{Resultados}

As Tabelas II e III apresentam, para tráfego de entrada e saída de diferentes pontos de acesso (PA), o resultado de estimação dos parâmetros estatísticos $\left(m, \sigma^{2}\right.$ e $\left.H\right)$ e aos parâmetros $\alpha$ e $\gamma$ dos traces. No estudo, foram considerados os tráfegos de entrada e de saída de 3 pontos de acesso selecionados aleatoriamente com $\alpha>1,7$. Para a estimação do parâmetro $H$, foi utilizada a ferramenta SET [26]. Os resultados de estimação foram obtidos pelo método baseado em Wavelets [27]. Note que o parâmetro de $H$ é adequadamente reproduzido pelos modelos estudados neste trabalho. Pode-se observar que os pontos de entrada dos prédios MBldg e LBldg apresentam $\alpha<2$ ou característica de alta impulsividade.

$\mathrm{Na}$ geração das amostras do modelo $\mathrm{fBm}$, foram utilizados os valores de $H, m$ e $\sigma^{2}$ dos traces originais da rede sem fio. O modelo Pseudo-MMPP define $\rho(1)$ como parâmetro de entrada. Entretanto, o algoritmo de Andersen e Nielsen não demonstrou resultados consistentes para valor de $\rho(1)$ maior que 0,7 . Ou seja, o algoritmo retornou valores não válidos para os parâmetros dos processos MMPP. Por isso, neste trabalho foi adotado o primeiro valor de $\rho($.) menor que 0,7 encontrado na análise do valor de $\rho(1)$ do trace correspondente. Por exemplo, para o trace do tráfego de entrada do ponto de acesso SBLdg foi utilizado $\rho(10)=0,663$.

As Figuras 3 e 4 ilustram os resultados de HTD das amostras geradas com os modelos fBm, $M / G / \infty$ e Pseudo-MMPP para os prédios SBldg, MBldg e LBldg. É possível fazer algumas observações sobre os modelos a partir da comparação dos resultados de HTD com os os resultados apresentados nas Tabelas II e III.

\begin{tabular}{|l|c|c|c|c|c|}
\hline Seqüências & $\hat{m}$ & $\hat{\sigma}^{2}$ & $\hat{H}$ & $\hat{\alpha}$ & $\hat{\gamma}$ \\
\hline \multicolumn{6}{|c|}{ SBldg } \\
\hline Original & 165,02 & 15058,87 & 0,791636 & 2,00 & 75,48 \\
\hline fBm & 166,41 & 11919,04 & 0,788906 & 2,00 & 83,87 \\
\hline$M / G / \infty$ & 129,34 & 114300,04 & 0,781989 & 1,57 & 60,24 \\
\hline Pseudo-MMPP & 149,95 & 2650,37 & 0,764429 & 2,00 & 41,41 \\
\hline \multicolumn{7}{|c|}{ MBldg } \\
\hline Original & 415,42 & 116408,30 & 0,763380 & 1,88 & 192,44 \\
\hline fBm & 427,52 & 90042,14 & 0,771028 & 2,00 & 234,56 \\
\hline$M / G / \infty$ & 544,89 & 256678,56 & 0,816508 & 2,00 & 376,35 \\
\hline Pseudo-MMPP & 403,21 & 3441,12 & 0,758128 & 2,00 & 50,32 \\
\hline \multicolumn{7}{|c|}{ LBldg } \\
\hline Original & 918,06 & 355184,40 & 0,763575 & 1,76 & 277,14 \\
\hline fBm & 923,13 & 301247,18 & 0,762145 & 2,00 & 409,37 \\
\hline$M / G / \infty$ & 1216,87 & 647723,05 & 0,765305 & 2,00 & 627,42 \\
\hline Pseudo-MMPP & 912,27 & 5737,78 & 0,767378 & 2,00 & 63,95 \\
\hline
\end{tabular}

TABELA II

ESTIMAÇÃO PARÂMETROS DO TRÁFEGO DE ENTRADA COM 8639 AMOSTRAS

\begin{tabular}{|l|c|c|c|c|c|}
\hline Seqüências & $\hat{m}$ & $\hat{\sigma}^{2}$ & $\hat{H}$ & $\hat{\alpha}$ & $\hat{\gamma}$ \\
\hline \multicolumn{7}{|c|}{ SBldg } \\
\hline Original & 109,65 & 1547,79 & 0,796545 & 2,00 & 30,93 \\
\hline fBm & 108,57 & 1430,53 & 0,776617 & 2,00 & 26,73 \\
\hline$M / G / \infty$ & 122,26 & 1953,14 & 0,757701 & 1,24 & 11,15 \\
\hline Pseudo-MMPP & 171,49 & 1538,07 & 0,747266 & 2,00 & 31,97 \\
\hline \multicolumn{7}{|c|}{ MBldg } \\
\hline Original & 249,28 & 14010,31 & 0,743784 & 2,00 & 77,58 \\
\hline fBm & 248,62 & 12957,04 & 0,740492 & 2,00 & 82,29 \\
\hline$M / G / \infty$ & 310,39 & 23282,77 & 0,829044 & 2,00 & 120,56 \\
\hline Pseudo-MMPP & 217,91 & 19858,99 & 0,775247 & 2,00 & 111,65 \\
\hline \multicolumn{7}{|c|}{ LBldg } \\
\hline Original & 587,90 & 48436,85 & 0,763999 & 2,00 & 132,09 \\
\hline fBm & 584,42 & 45491,01 & 0,752306 & 2,00 & 150,94 \\
\hline$M / G / \infty$ & 689,49 & 102748,04 & 0,804526 & 1,20 & 81,84 \\
\hline Pseudo-MMPP & 580,00 & 1799,37 & 0,721105 & 2,00 & 33,55 \\
\hline
\end{tabular}

TABELA III

ESTIMAÇÃO PARÂMETROS DO TRÁFEGO DE SAÍDA COM 8639 AMOSTRAS

O fBm é um modelo Gaussiano, e portanto, só é capaz de gerar amostras com $\alpha=2$. Para os traces que originalmente possuem $\alpha=2$, o fBm obteve uma boa aproximação para o parâmetro $\gamma$ e por isso, consegue uma boa aproximação da HTD do trace original.

O modelo $M / G / \infty$ apresentou valores de variância maior que o valor do arquivo original. Isto é explicado pelo tamanho escolhido para geração das amostras. Neste estudo, foram geradas sequiências de 8639 amostras para todos os modelos de forma a se obter o mesmo tamanho do trace original. Nos testes realizados, o $M / G / \infty$ apresentou melhor aproximação dos parâmetros estatísticos para arquivos com pelo menos 32000 amostras. Entretanto, é interessante notar que o $M / G / \infty$ apresenta sempre a cauda próxima dos traces 


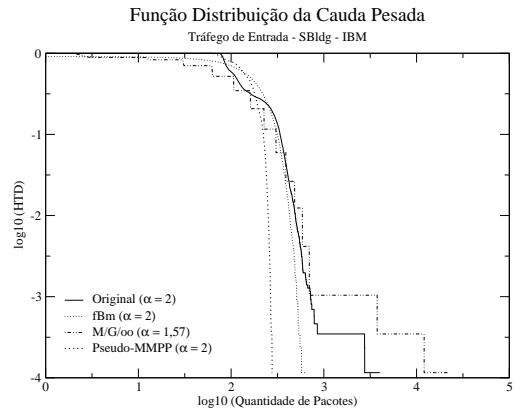

(a)

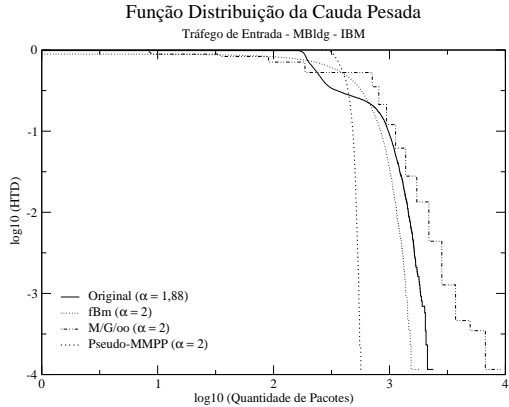

(b)

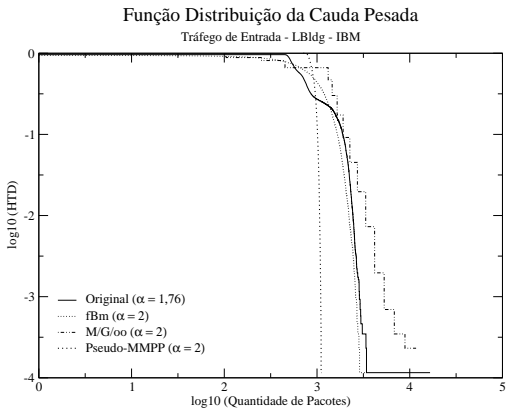

(c)

Fig. 3. Tráfego de entrada dos prédios (a) SBldg, (b) MBldg e (c) LBldg.

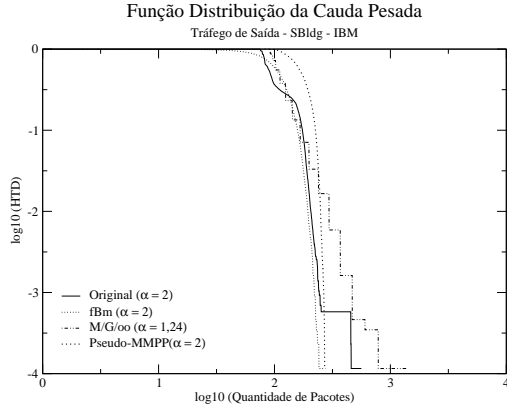

(a)

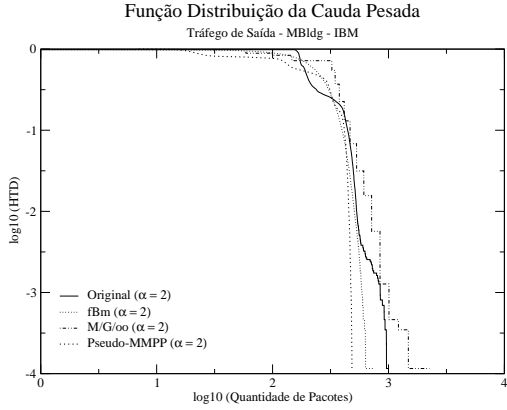

(b)

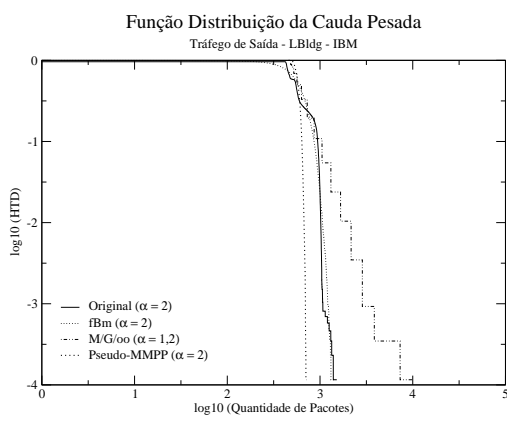

(c)

Fig. 4. Tráfego de saída dos prédios (a) SBldg, (b) MBldg e (c) LBldg.

originais, mesmo quando o modelo apresenta valor de $\alpha$ maior (por exemplo, o trace de entrada do prédio MBldg). Isto é explicado pelo fato de que este modelo define a distribuição $G$ de cauda pesada (Pareto) de forma a obter a dependência de longo alcance.

O modelo Pseudo-MMPP não consegue modelar adequadamente a HTD para a maioria dos traces estudados. Isto se deve ao fato do modelo Pseudo-MMPP, por definição, conseguir apenas uma aproximação para a função autocorrelação do trace original. Ou seja, o algoritmo de Andersen e Nielsen [16] ignora as características da HTD do trace original quando calcula os parâmetros básicos do modelo. Note que em apenas dois casos, tráfegos de saída dos prédios SBldg e MBldg, o modelo consegue representar de forma adequada os parâmetros estatísticos e os parâmetros $\alpha$ e $\gamma$. Nestes dois exemplos, a HTD do modelo Pseudo-MMPP está bem próxima das HTDs do traces original e do modelo $\mathrm{fBm}$.

A importância do parâmetro $\alpha$ ou impulsividade do tráfego de redes sem fio, pode ser examinada através do comportamento de uma fila infinita $(G / D / 1)$ com distribuição $G$ caracterizada pelos modelos fBm, $M / G / \infty$ e Pseudo-MMPP. Suponha que os traces coletados nos pontos de acesso do prédio SBldg sejam redirecionados para um servidor de pacotes com uma fila infinita. Neste caso, tanto o tráfego de entrada quanto o tráfego de saída do ponto de acesso são considerados como tráfego de entrada para o servidor de pacotes. A taxa de serviço utilizada é igual a $170 \%$ da taxa média de chegadas de pacotes. Este valor foi escolhido de forma a garantir que a transmissão do número médio de pacotes correspondesse a ocupação de $60 \%$ do enlace ou utilização do servidor.

A Figura 5 mostra os resultados do comportamento da HTD do número de pacotes na fila para os traces originais, os diferentes processos de chegada e graus de impulsividade. Observando-se as curvas, pode-se verificar que a HTD do tráfego gerado pelo modelo $M / G / \infty$ se alonga além das HTDs dos tráfegos gerados pelos outros modelos e pelos traces originais. Este resultado é definido pelo valor do parâmetro $\alpha$ das amostras geradas pelo $M / G / \infty$. O parâmetro $\alpha$ apresenta valores 1,57 (entrada SBldg) e 1,24 (saída SBldg) para o $M / G / \infty$ e valor 2 para os outros modelos. Portanto, a cauda do $M / G / \infty$ é, como esperado, mais longa. Para as demais seqüências amostrais com $\alpha=2$, apresentadas na Figura 5, a diferença na HTD se deve as distintas funções densidade de probabilidade dos modelos.

Os resultados apresentados nesta seção demonstraram a necessidade de novas pesquisas de forma a propor teorias de avaliação de desempenho de sistemas e redes de comunicação com características de impulsividade.

\section{Conclusões}

Neste artigo foi apresentado uma análise da impulsividade do tráfego em redes sem fio com tecnologia IEEE 802.11. Para a estimação da impulsividade presente, foi utilizado o método de McCulloch. A representação da impulsividade foi examinada e discutida considerando-se diferentes modelos estocásticos de cauda-pesada (fBm, $M / G / \infty$ e Pseudo-MMPP). Os resultados de estimação demonstraram que o tráfego de redes sem fio apresenta alto grau de impulsividade.

De forma geral, os modelos $\mathrm{fBm}$ e $M / G / \infty$ obtiveram o melhor resultado de representação da impulsividade. Resultados de medidas de desempenho em filas também foram 

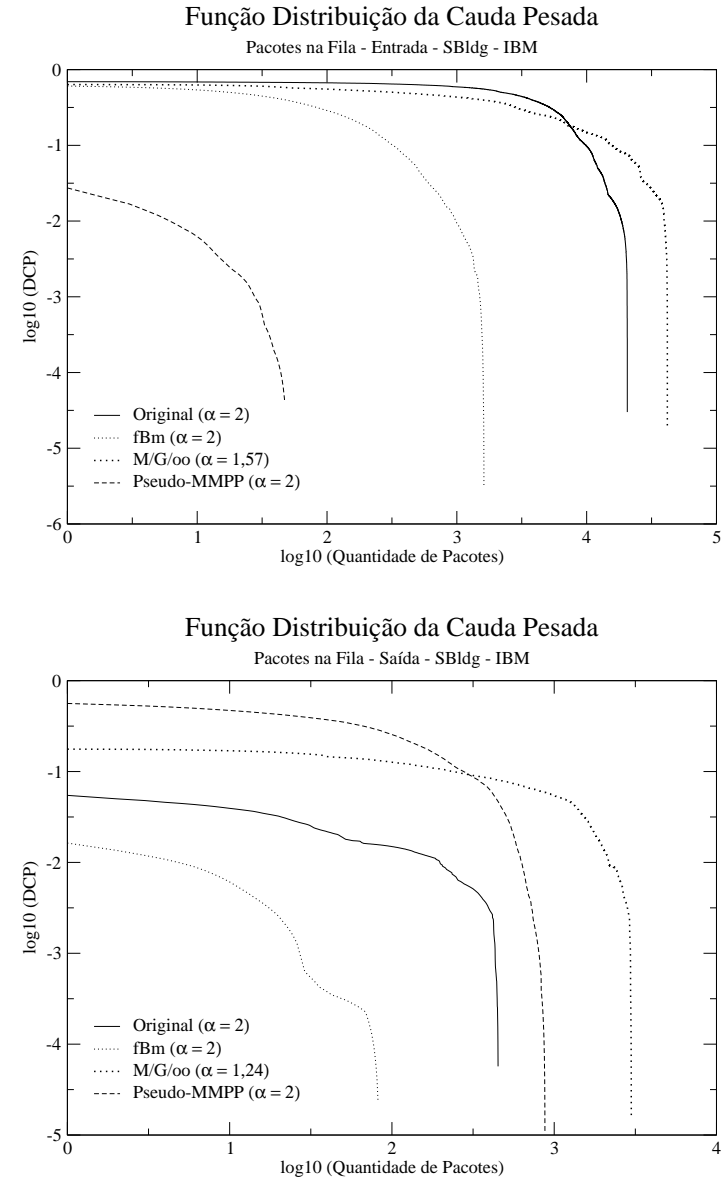

(b)

Fig. 5. Tráfego de entrada (a) e saída (b) do prédio SBldg.

determinados para processos de chegada com caracterísitcas de impulsividade representada por distribuições com cauda pesada.

Este estudo demonstrou que a estimação de parâmetros dos processos de cauda-pesada é ainda um desafio para a pesquisa. Além disso, devem ser elaborados novos estudos da teoria de simulação e modelagem de forma a prover melhor precisão nos resultados de medidas de desempenho para sinais com características de impulsividade. Também pode-se ressaltar a necessidade de obtenção de uma maior quantidade de traces representativos do tráfego de usuários e conexões de redes sem fio.

\section{REFERÊNCIAS}

[1] IEEE, " $802.11 \mathrm{~b} / \mathrm{d} 3.0$ wireless lan medium access control (mac) and physical layer (phy) specification," August 1999.

[2] "http://ramp.ucsd.edu/pawn/sigcomm-trace," 2001.

[3] "http://cmc.cs.dartmouth.edu/data," 2002.

[4] "http://nms.lcs.mit.edu/ mbalazin/wireless," 2002.

[5] A. Balachandran, G. M. Voelker, P. Bahl, and P. V. Rangan, "Characterizing user behavior and network performance in a public wireles lan," ACM SIGMETRICS'02, June 2002.

[6] W. E. Leland, M. S. Taqqu, W. Willinger, , and D. Wilson, "On the self-similar nature of ethernet traffic," IEEE/ACM Transactions on Networking, vol. 2, pp. 1-15, February 1994.

[7] D. S. Sharp, N. Cackov, N. Laskovic, Q. Shao, and L. Trajkovic, "Analysis of public safety traffic on trunked land mobile radio systems," IEEE Journal on Selected Areas in Communications, vol. 22, pp. 11971205, September 2004.
[8] M. Toscano, M. Diniz, and R. Coelho, "Caracterização do tráfego de redes locais sem fio baseada em processos pseudo-markovianos e fractais," Anais do XXII Simpósio Brasileiro de Telecomunicações, pp. 487-492, 2005.

[9] F. Hernández-Campos and M. Papadopouli, "A comparative measurement study of the workload of wireless access points in campus networks," in 16th International Symposium on Personal Indoor and Mobile Radio Communications, September 2005.

[10] O. Tickoo and B. Sikdar, "On the impact of ieee $802.11 \mathrm{mac}$ on traffic characteristics," IEEE Journal on Selected Areas in Communications, vol. 21, pp. 189-203, Feb 2003.

[11] G. Samorodnitsky and M. Taqqu, Stable Non-Gaussian Random Processes: Stochastic Models and Infinite Variance. London: Chapman \& Hall, 1994.

[12] C. Nikias and M. Shao, Signal Processing with Alpha-Stable Distributions and Applications. New York: Wiley, 1995.

[13] M. Shao and C. Nikias, "Signal processing with fractional lower order moments: Stable processes and their applications," Proceedings of the IEEE, vol. 81, no. 7, pp. 986-1010, 1993.

[14] B. Mandelbrot and J. Van Ness, "Fractional brownian motions, fractional noises and applications," SIAM Review, vol. 10, pp. 422-437, October 1968.

[15] M. Krunz and A. Makowski, "Modeling video traffic using $\mathrm{m} / \mathrm{g} / \infty$ input processes: A compromise between markovian and lrd models," IEEE J. Select. Areas Commun., vol. 16, pp. 733-748, June 1998.

[16] A. Andersen and B. Nielsen, "A markovian approach for modeling packet traffic with long-range dependence," IEEE JSAC, vol. 16, pp. 719732, June 1998.

[17] I. Lee and A. Fapojuwo, "Estimating heavy-tails in long-range dependent wireless traffic," Vehicular Technology Conference, vol. 4, pp. 2132 2136, May 2005.

[18] H. J. McCulloch, "Simple consistent estimators of stable distribution parameters," Communications in Statistics, vol. 15, no. 4, pp. 11091136, 1998.

[19] S. kogon and D. Williams, "On the characterization of impulsive noise with $\alpha$-stable distributions using fourier techniques," 29th Asilomar Conference Signals, Syst. and Comp., 1995.

[20] X. Ma and L. Nikias, "Parameter estimation and blind channel identification of impulsive signal environments," IEEE Trans. on Signal Processing, vol. 43, pp. 2884-2897, 1995.

[21] G. Tsihrintzis and L. Nikias, "Fast estimation of parameters of alphastable impulsive interference," IEEE Trans. on Signal Processing, vol. 44, pp. 1492-1503, 1996.

[22] S. Bates and S. McLaughlin, "The estimation os stable distribution parameters from teletraffic data," IEEE Transactions on Signal Processing, vol. 48, pp. 865-870, March 2000.

[23] M. Crovella and L. Lipsky, Simulations with Heavy-Tailed Workloads. Self-similar Network Traffic and Performance Evaluation: Chapter 3. John Wiley and Sons, 2000.

[24] M. Balazinska and P. Castro, "Characterizing mobility and network usage in a corporate wireless local-area network," Proc. of The First International Conference on Mobile Sistems (MobiSys), 2003.

[25] I. Norros, "On the use of fractional brownian motion in theory of connectionless networks," IEEE JSAC, vol. 13, pp. 953-962, August 1995.

[26] L. Zão, J. Filho, A. Pereira, M. Diniz, and R. Coelho, "Set (scaling estimation tool): Uma ferramenta gráfica de estimação e análise de sistemas com características de dependência temporal," 24 Simpósio Brasileiro de Redes de Computadores, Maio 2006.

[27] M. Roughan, D. Veith, and P. Abry, "Real-time estimation of the parameters of long-range dependence," IEEE/ACM Transactions on Networking, vol. 8, pp. 467-478, August 2000. 